\title{
Rasgos diferenciales del temperamento y el carácter en un grupo de alcoholicos vs poblacion general
}

\author{
Julián Mateos Agut*; Manuel Mateos Agut** \\ (*) Psicólogo. ARBU (Asociación de Alcohólicos Rehabilitados de Burgos). \\ (**) Psiquiatra-Coordinador. Hospital de Día del Servicio de Psiquiatría del Hospital "Divino Valles" de Burgos.
}

Enviar correspondencia a:

ARBU (Asociación de Alcohólicos Rehabilitados de Burgos). C/ Fray Justo Pérez de Urbel n 7 bajo. 09006-Burgos. Teléfono (947) 241924. Email: arbu00@terra.es

Recibido: 11 de marzo de 2003 Aceptado: 11 de abril de 2005

\section{RESUMEN}

Los autores de este estudio analizan los rasgos o características de personalidad entre dos grupos: pacientes alcohólicos según criterios DSM-IVR y población normal. Se aplica el Inventario de Temperamento y Carácter (T.C.I. de Cloninger) en ambas muestras y se analiza los resultados. El objetivo es identificar diferencias significativas entre los rasgos de personalidad de los dos grupos. Los resultados muestran los varones alcohólicos, respecto a las mujeres alcohólicas, son más cooperativos y autodirectivos, mientras que ellas puntúan más alto en Evitación del Riesgo y en Dependencia de la Recompensa. En relación con los varones control el varón alcohólico puntúa más alto en Búsqueda de Novedades, en Evitación del Riesgo y en Autotrascendencia; y es menos autodirectivo y cooperativo. Observamos que la mujer alcohólica es menos cooperativa y autodirectiva que la mujer control, en cambio tiende a evitar el riesgo y a ser más autotrascendente.

Palabras-clave: Rasgos de Personalidad, Alcoholismo, TCl, Búsqueda de Novedades, Evitación del Riesgo, Autodirección, Dependencia de la Recompensa, Cooperación.

\section{ABSTRACT}

In this study, the authors analyse the features or characteristics of personality between two groups: alcoholic patients diagnosed according the DSM-IVR and normal population. In both samples, the Temperament and Character Inventory (Cloninger's TCI) is used and the results are analyzed. The objective is to identify relevant differences in personality features between the two groups. The results show that male alcoholics, in comparison with female alcoholics, are more cooperative and self-directional and female alcoholics have a higher score in Risk Avoiding and in Dependence on Reward. Compared with the male control, male alcoholics have a higher score regarding Novelty Seeking and Risk Avoiding and in Selftranscendence; and are less self-directed and cooperative. We observe that female alcoholic are less cooperative and self-directed than female control, however they have a greater tendency to avoid risk and to be more self-transcendental.

Keywords: Personality Features, Alcoholism, TCl, Novelty Seeking, Risk avoiding, Self-direction, Dependence on Reward, Cooperation.

\section{INTRODUCCIÓN}

$\mathbf{E}$ estudio de la personalidad sigue siendo un campo controvertido tanto desde la psiquiatría, como desde la psicología. En la actualidad, se siguen elaborando gran número de investigaciones sobre la personalidad basadas en modelos teóricos diferentes (biológico, psicológico, social) que explican la personalidad desde variados puntos de vista, a veces confluyentes y otras veces no, pero todos ellos nada desechables.

A lo largo de los últimos años distintas investigaciones clínicas avalan la hipótesis de que existen ras- gos característicos que definirían la personalidad del alcohólico, mientras que otros autores, no menos numerosos, defienden que el conjunto de rasgos observados podrían ser debidos a los efectos directos del alcohol sobre la biología, la capacidad operacional y relacional del sujeto y su entorno, pero que remitirían tras suspenderse el consumo de alcohol (Casariego, Morera, Henry, Valenciano, Bello y Cuesta, 1995). Estos autores añaden que un trastorno de personalidad puede cursar con síntomas de alcoholismo, pero eso no significa que esos rasgos definan una personalidad alcohólica tipo.

Sin embargo parece haber acuerdo en que los Trastornos psiquiátricos (depresión, trastornos de ansie- 
dad y trastornos de la personalidad) tienen mayor prevalencia entre población alcohólica que la población general (Modesto-Lowe y Kranzler, 1999; Mateos, Sanz, Palomo, García, Merino y De la Gándara, 2004).

Determinar si existen diferencias significativas en los rasgos de personalidad de pacientes alcohólicos y la población general, es el objetivo de este estudio, que contribuiría a aclarar algunos puntos de la discusión. Para la elaboración de este estudio nos hemos basado en un modelo mixto, el Modelo Psicobiológico de la Personalidad de Robert C. Cloninger (1993,1994,1997), que integra la personalidad en los componentes: biológico y psicológico (Mateos, Ruiz y De la Gándara, 2003).

Cloninger recupera en su modelo los conceptos de Temperamento (parte de la personalidad fundamentalmente heredada y dependiente de módulos neuroquímicos) y el Carácter (parte predominantemente aprendida durante el desarrollo en la interacción con el medio), y postula que el Temperamento esta constituido por tres dimensiones de la personalidad -Búsqueda de novedades (NS), Evitación del riesgo (HA), y Dependencia de la recompensa (RD)-, cada una de las cuales estaría definida según un neurotransmisor específico presente en las vías neuronales del sistema cerebral. Posteriormente identificó un cuarto factor que denominó Persistencia $(P)$. Estas dimensiones se pueden presentar en diferentes combinaciones en los distintos seres humanos, dando cuenta, por tanto, de la organización funcional que subyace en cada individuo.

El carácter, en cambio, estaría organizado en tres dimensiones, más determinadas por la interacción con el entorno, que describen los autoconceptos sobre uno mismo (Autodirección (S)); sobre la relación con los otros (Cooperación (C)), y con el mundo entendido como un todo (Autotrascendencia (ST)).

Para algunos autores que analizan la personalidad desde este modelo, la población alcohólica se separa de la población No clínica en algunas de las dimensiones del TCl. Las características principales del alcoholismo serían: la alta puntuación en Búsqueda de Novedades ( $\uparrow$ N), Baja Evitación del Riesgo ( $\downarrow H A)$ y la baja puntuación en Autodirección ( $\downarrow S)$ (Kampov-Polevoy, Garbutt, Davis y Janowsky, 1998). Esta baja Autodirección indicaría una mayor probabilidad de presentar trastornos de la personalidad, y describe una falta de control de la propia conducta (Basiaux et al., 2001).

En su aproximación a la personalidad del alcohólico Cloninger (1987), distinguió dos tipos de alcoholismo: tipo I y tipo II. El alcoholismo tipo I (Belloch y Sandín, 1995) se caracterizaría por una tendencia a evitar el dolor y hacia la obtención de recompensas; mientras que el alcoholismo tipo II, tendría como características principales la aparición de conductas antisociales, la búsqueda de novedades y nuevas sensaciones, y una mayor influencia genética, al menos en varones. Este modelo fue refutado por otros estudios (Pomerlau, Pomerleau, Flessland y Basson, 1992) que encuentra que los alcohólicos tipo I se caracterizarían por tener bajas Búsqueda de Novedades y altas Evitación del Riesgo y Dependencia de la Recompensa ( $\downarrow N S$; $\uparrow H A$; $\uparrow R D)$, siendo tan frecuente en los varones como en las mujeres; mientras que el alcoholismo tipo II, más característico de los varones, tiene alta Búsqueda de Novedades y bajas Evitación del Riesgo y Dependencia de la Recompensa ( $\uparrow N S ; \downarrow H A ; \downarrow R D)$.

Otros autores (Weijers, Wiesbeck y Boening, 1999) clasifican el alcoholismo en función de dos pares de variables: Evitación del Riesgo - Autodirección y Dependencia de la Recompensa - Autotrascendencia, haciendo el modelo más operativo para la intervención.

En algunos trabajos, en los que se controló la variable Edad de Inicio del Consumo de Alcohol para discriminar entre los tipos de alcoholismo, se pudo determinar que cuanto ésta era más precoz, más severa era la psicopatología: más graves los problemas relacionados con el consumo de alcohol y los trastornos de conducta. De manera que los rasgos de personalidad antisocial se aproximarían a las características del alcoholismo tipo II, cuanto más precoz es la edad de inicio de consumo de alcohol (Johnson, Cloninger, Roache, Bordnick y Ruiz, 2000).

La Búsqueda de Novedades ha sido una variable muy estudiada en los últimos años como lo muestra la gran cantidad de trabajos existentes. Los autores Ilegan a concluir que altas puntuaciones en esta variable ( $\uparrow$ N) tendría un valor predictivo de varios factores:

- Inicio precoz del consumo, con alta probabilidad de aparición de problemas conductuales (Cloninger, Sigvardsson, Svrakic y Przybeck, 1995),

- Mala evolución (recaídas frecuentes) en el tratamiento (Cloninger, et al. 1995; Fallgatter, Weijers, Wiesbeck, Boening y Strik, 1998), que también pudo ser observada en una población de mujeres alcohólicas (Kitamura, Kijma, Sakamoto, Tomoda, Suzuki y Kazama, 1999)

- Conductas antisociales, asociadas a altos índices de criminalidad en alcohólicos (Cloninger, et al. 1995; Howard, Kivlahan y Walker, 1997)

- Trastorno Disocial de la Personalidad (Sander et al., 1998)

- También se ha relacionado con el genotipo A1/A2 en sujetos alcohólicos (Thome et al., 1999).

La interacción entre Búsqueda de Novedades y los factores antes señalados parece ser independientes del sexo (Meszaros, Willinger, Fischer, Schonbeck, Aschaner, 1996). 
Por tanto, analizar la búsqueda de Novedades de nuestros pacientes podría ser útil para el despistaje psicopatológico, pues se asocia con el alcoholismo tipo II y con el Trastorno de Personalidad Antisocial (Cloninger et al., 1995).

Por otra parte, Puntuaciones bajas en Evitación del Riesgo ( $\downarrow$ HA) contribuyen también a predecir el inicio temprano del consumo de sustancias y el abandono precoz del tratamiento (Schmidt, Dufeu, Kuhn y Rommelspacher, 1994; Willis, Vaccaro y Mcnamara, 1994), y se asocia, en el caso de pacientes alcohólicos, a un posible diagnóstico de Trastorno Disocial de la Personalidad y de Alcoholismo Tipo II (Sander et al., 1998).

En este estudio, intentaremos describir rasgos de personalidad de un grupo de alcohólicos/as, mediante la aplicación del $\mathrm{TCl}$ que compararemos con los de un grupo de población normal no alcohólica

\section{MATERIAL Y MÉTODO}

\section{MUESTRA:}

Para realizar el presente estudio, obtuvimos 2 grupos equiparados en edad y sexo:
- Un grupo de población alcohólica N = 145, de ambos sexos, varones (VA) y mujeres (MA), diagnosticados de Trastorno por Dependencia de Alcohol según criterios DSM-IV-R, que fueron admitidos a tratamiento en la Asociación de Alcohólicos Rehabilitados de Burgos (ARBU) y en el Hospital de Día del Servicio de Psiquiatría del Hospital Divino Valles de Burgos durante el periodo Enero 2000 a Diciembre del 2002

- Un grupo Control de población no clínica, $\mathrm{N}=$ 145, obtenida para la baremación del TCl (Mateos y De la Gándara, 2001) entre familiares del personal del Hospital Divino Valles de Burgos y Centros Universitarios de Burgos y Valladolid.

- La corrección de los cuestionarios se realizó por medio de la Aplicación Informática para el Tratamiento de Datos, Corrección del Test y Emisión de Informes (Mateos y De la Gándara, 2001)

$\mathrm{EITCl}$ se administró tras cuatro semanas de abstinencia alcohólica, desde el inicio del tratamiento.

Para controlar la variable Sexo se han dividido las muestras en cuatro grupos igualados en número de sujetos y elegidos al azar con edades comprendidas entre 21 y 76 años, como se muestra en la tabla I:

\begin{tabular}{|lcccc|}
\hline \multicolumn{5}{c|}{ TABLA I. EDAD GRUPOS DE ESTUDIO } \\
N & $\begin{array}{c}\text { VARONES } \\
\text { ALCOHÓLICOS (VA) }\end{array}$ & $\begin{array}{c}\text { MUJERES } \\
\text { ALCOHÓLICAS (MA) }\end{array}$ & $\begin{array}{c}\text { VARONES } \\
\text { CONTROLES (VC) }\end{array}$ & $\begin{array}{c}\text { MUJERES } \\
\text { CONTROLES (MC) }\end{array}$ \\
Media & 106 & 39 & 106 & 39 \\
Mediana & 41,28 & 42,69 & 41,76 & 42,54 \\
Moda & 40,00 & 42,00 & 40,00 & 42,00 \\
Desv. Típica & 32 & 38 & 40 & 38 \\
Mínimo & 9,750 & 9,362 & 11,681 & 9,290 \\
Máximo & 21 & 26 & 21 & 26 \\
\hline
\end{tabular}

- Alcohólicos (A):

1. Varones alcohólicos (VA), son una muestra de $\mathrm{N}=106$, cuya edad media es de 41,2 años.

2. Mujeres Alcohólicas (MA), son una muestra de $\mathrm{N}=39$ y su edad media es 42,6 años.

- Población No Clínica (C)

1. Varones controles (VC), son una muestra de $\mathrm{N}=$ 106 de población voluntaria que acepto libremente participar en el estudio, cuya edad media es de 41,7 años.
2. Las mujeres controles (MC), son una muestra de $\mathrm{N}=39$ y su edad media es 42,5 años.

Desgraciadamente, no se ha podido controlar la variable Nivel de Estudios en las muestras de Alcohólicos y Controles, si bien, el extracto de ambas muestras era similar.

\section{DESCRIPCION DEL INSTRUMENTO DE MEDICION:}

Para medir la personalidad Cloninger elaboró un inventario TCl (del temperamento y del carácter), con las siguientes escalas y subescalas: 
Tabla II Escala y subescalas del cuestionario de personalidad de Cloninger (TCl)

\begin{tabular}{|c|c|c|c|c|c|}
\hline $\begin{array}{l}\text { ESCALA } \\
\text { Temperamento }\end{array}$ & $\begin{array}{c}\text { Subescala } \\
1\end{array}$ & $\begin{array}{c}\text { Subescala } \\
2\end{array}$ & $\begin{array}{c}\text { Subescala } \\
3\end{array}$ & $\begin{array}{c}\text { Subescala } \\
4\end{array}$ & $\begin{array}{c}\text { Subescala } \\
5\end{array}$ \\
\hline $\begin{array}{l}\text { Búsqueda de } \\
\text { Novedades (NS) }\end{array}$ & $\begin{array}{c}\text { Rigidez/ } \\
\text { Búsqueda de sensac. }\end{array}$ & $\begin{array}{l}\text { Reflexión/ } \\
\text { Impulsividad }\end{array}$ & $\begin{array}{c}\text { Reserva/ } \\
\text { Extravagancia }\end{array}$ & $\begin{array}{c}\text { Orden/ } \\
\text { Desorden }\end{array}$ & \\
\hline $\begin{array}{l}\text { Evitación del Riesgo } \\
(\mathrm{HA})\end{array}$ & Optimismo/ Ansiedad & $\begin{array}{l}\text { Confianza/ } \\
\text { Miedo }\end{array}$ & $\begin{array}{l}\text { Gregario/ } \\
\text { Tímido }\end{array}$ & $\begin{array}{l}\text { Vigor/ } \\
\text { Fatiga }\end{array}$ & \\
\hline $\begin{array}{l}\text { Dependencia de la } \\
\text { Recompensa (RD }\end{array}$ & $\begin{array}{l}\text { Independencia/ } \\
\text { Dependencia }\end{array}$ & & $\begin{array}{l}\text { Desapego/ } \\
\text { apego }\end{array}$ & $\begin{array}{l}\text { Insensible/ } \\
\text { Sentimental }\end{array}$ & \\
\hline Persistencia (P) & Falta/ausencia & & & & \\
\hline Carácter & 1 & 2 & 3 & 4 & 5 \\
\hline Autodirección (S) & $\begin{array}{l}\text { Proy. Culpa/ } \\
\text { Responsable }\end{array}$ & $\begin{array}{l}\text { No metas/ } \\
\text { Determinación }\end{array}$ & $\begin{array}{c}\text { Inercia/ } \\
\text { Sin recursos }\end{array}$ & $\begin{array}{c}\text { Competir/ } \\
\text { Autoaceptar }\end{array}$ & $\begin{array}{l}\text { Malo/ Buen } \\
\text { Hábito }\end{array}$ \\
\hline Cooperación ( C ) & $\begin{array}{l}\text { Intolerancia/ } \\
\text { Aceptación }\end{array}$ & $\begin{array}{l}\text { Insensibilidad/ } \\
\text { Empatía }\end{array}$ & $\begin{array}{l}\text { Egoísmo/ } \\
\text { Ayuda }\end{array}$ & $\begin{array}{l}\text { Veganza/ } \\
\text { Compasión }\end{array}$ & $\begin{array}{l}\text { Oportunismo/ } \\
\text { Principios }\end{array}$ \\
\hline Autotranscendencia (ST) & $\begin{array}{c}\text { Cohibición/ } \\
\text { Autoabandono }\end{array}$ & $\begin{array}{c}\text { Autodiferencia/ } \\
\text { Identidad Transpersonal }\end{array}$ & $\begin{array}{l}\text { Materialismo/ } \\
\text { Espiritualidad }\end{array}$ & & \\
\hline
\end{tabular}

\section{PROCEDIMIENTO:}

Para la investigación se realizó un estudio comparativo entre una muestra de pacientes alcohólicos (A) y un grupo control no alcohólico (C) a los que se administró el TCl (Inventario del Temperamento y el Carácter) de Robert C. Cloninger, inventario ya validado en España (Gutiérrez, Torrens, Martín-Santos, Sangorrin, Pérez y Salamero, 2001), al mes del inicio del tratamiento. Posteriormente, se compararon los distintos subgrupos entre sí para controlar la variable Sexo: grupo 'VA' (varón alcohólico) vs grupo 'MA' (mujer alcohólica); grupo 'VC' (varón control) vs ' $M C^{\prime}$ ' (mujer control); grupo 'VA' vs grupo 'VC'; y Grupo 'MA' vs grupo 'MC'

Los cálculos estadísticos se realizaron con el apoyo del programa estadístico SPSS. Se compararon las diferencias en las medias obtenidas en cada grupo, mediante la prueba t, para cada una de las escalas y subescalas, y los resultados que se muestran en las tablas II, III, IV y V son los que resultaron estadísticamente significativos.

Hay que señalar que en las tablas II, IV y V puede observarse que en algunas subescalas de los grupos de varones, aparecen 105 casos (siendo la muestra total de Alcohólicos y controles de 106). Este hecho obedece a que cuando el sujeto deja sin contestar una pregunta en el cuestionario, queda invalidada toda la subescala y posteriormente toda la escala. Dado que se trataba tan solo de 2 sujetos y que el resto de las respuestas eran válidas, optamos por incluirlos en la muestra.

\section{RESULTADOS}

En los siguientes recuadros exponemos los resultados más significativos de nuestro estudio. En primer lugar se muestra las diferencias significativas encontradas entre los Varones Alcohólicos y los Varones Control (tabla III):

DIFERENCIAS ENTRE VARONES ALCOHÓLICOS Y VARONES CONTROLES

- Los varones alcohólicos puntúan más alto en Búsqueda de Novedades ( $\uparrow N S$ ): y en sus subescalas: Impulsividad, Extravagancia y Desorden, lo que indicaría una tendencia a guiarse por la emociones y los estados de ánimo ( $\uparrow N S 2$ ), a ser poco precavidos y previsores (por ejemplo en el control del dinero) ( $\uparrow N S 3$ ) y a preferir la ausencia de reglas ( $\uparrow N S 4)$. Al contrario los controles serían más reflexivos y menos emocionales.

- El varón alcohólico tienen más altas las puntuaciones en Evitación del Riesgo ( $\uparrow H A$ ): lo que indica una mayor Ansiedad Anticipatoria y pesimismo ( $\uparrow H A 1$ ) y Fatigabilidad ( $\uparrow H A 4$ ), por lo que se sienten con menos energía y se recuperan más despacio que el varón control. 


\begin{tabular}{|c|c|c|c|c|c|c|c|}
\hline & NHIST & $\mathbf{N}$ & Media & Desviación típ. & $\mathbf{T}$ & gl & Sig. (bilateral) \\
\hline \multirow[t]{2}{*}{ NS2 } & VA & 105 & 4,5810 & 2,39302 & 2,217 & 209 & ,028 \\
\hline & VC & 106 & 3,8491 & 2,40156 & & & \\
\hline \multirow[t]{2}{*}{ NS3 } & VA & 106 & 5,5094 & 2,34316 & 4,133 & 210 &, 000 \\
\hline & VC & 106 & 4,2170 & 2,20823 & & & \\
\hline \multirow[t]{2}{*}{ NS4 } & VA & 106 & 4,3208 & 2,07271 & 2,615 & 210 & ,010 \\
\hline & VC & 106 & 3,6038 & 1,91575 & & & \\
\hline \multirow[t]{2}{*}{ NS } & VA & 105 & 19,7048 & 5,67709 & 3,317 & 209 & ,001 \\
\hline & VC & 106 & 17,0377 & 5,99670 & & & \\
\hline \multirow[t]{2}{*}{ HA1 } & VA & 106 & 5,5660 & 2,59310 & 3,867 & 210 &, 000 \\
\hline & VC & 106 & 4,1887 & 2,59345 & & & \\
\hline \multirow[t]{2}{*}{ HA4 } & VA & 106 & 4,0000 & 2,12020 & 3,848 & 210 &, 000 \\
\hline & VC & 106 & 2,9245 & 1,94544 & & & \\
\hline \multirow[t]{2}{*}{$\mathrm{HA}$} & VA & 106 & 18,5849 & 6,06452 & 3,248 & 209 & ,001 \\
\hline & VC & 105 & 15,8190 & 6,30443 & & & \\
\hline \multirow[t]{2}{*}{ RD3 } & VA & 106 & 4,1321 & 2,31794 & $-2,707$ & 210 & ,007 \\
\hline & VC & 106 & 4,9906 & 2,29905 & & & \\
\hline \multirow[t]{2}{*}{ RD4 } & VA & 106 & 2,7925 & 1,35729 & $-3,557$ & 210 &, 000 \\
\hline & VC & 106 & 3,4528 & 1,34612 & & & \\
\hline \multirow[t]{2}{*}{$\mathrm{RD}$} & VA & 106 & 14,1038 & 3,86419 & $-2,644$ & 210 &, 009 \\
\hline & VC & 106 & 15,4340 & 3,45038 & & & \\
\hline \multirow[t]{2}{*}{ S1 } & VA & 106 & 4,0000 & 2,23394 & $-7,281$ & 210 &, 000 \\
\hline & VC & 106 & 6,0377 & 1,82012 & & & \\
\hline \multirow[t]{2}{*}{ S2 } & VA & 106 & 4,4340 & 1,95189 & $-6,081$ & 210 &, 000 \\
\hline & VC & 106 & 5,9434 & 1,64942 & & & \\
\hline \multirow[t]{2}{*}{ S3 } & VA & 106 & 2,2170 & 1,40750 & $-5,642$ & 210 &, 000 \\
\hline & VC & 106 & 3,3491 & 1,51213 & & & \\
\hline \multirow[t]{2}{*}{ S5 } & VA & 105 & 5,8286 & 2,59607 & $-7,248$ & 209 &, 000 \\
\hline & VC & 106 & 8,3585 & 2,47343 & & & \\
\hline \multirow[t]{2}{*}{ S } & VA & 105 & 23,4476 & 7,14544 & $-7,956$ & 209 & ,000 \\
\hline & VC & 106 & 30,8774 & 6,40343 & & & \\
\hline \multirow[t]{2}{*}{ C1 } & VA & 106 & 5,6604 & 1,87661 & $-2,888$ & 210 &, 004 \\
\hline & VC & 106 & 6,3585 & 1,63409 & & & \\
\hline \multirow[t]{2}{*}{ C2 } & VA & 106 & 3,8019 & 1,46982 & $-4,315$ & 210 &, 000 \\
\hline & VC & 106 & 4,6604 & 1,42686 & & & \\
\hline \multirow[t]{2}{*}{ C4 } & VA & 106 & 7,0000 & 2,57460 & $-2,494$ & 210 & ,013 \\
\hline & VC & 106 & 7,8302 & 2,26136 & & & \\
\hline \multirow[t]{2}{*}{ C5 } & VA & 105 & 5,3619 & 1,37368 & $-6,591$ & 209 & ,000 \\
\hline & VC & 106 & 6,6132 & 1,38406 & & & \\
\hline \multirow[t]{2}{*}{ C } & VA & 105 & 26,6286 & 6,32799 & $-4,983$ & 209 &, 000 \\
\hline & VC & 106 & 30,6698 & 5,42081 & & & \\
\hline \multirow[t]{2}{*}{ ST1 } & VA & 106 & 6,5094 & 2,50426 & 4,181 & 210 &, 000 \\
\hline & VC & 106 & 5,0472 & 2,58708 & & & \\
\hline ST2 & VA & 106 & 4,5566 & 2,41850 & 4,113 & 210 &, 000 \\
\hline & VC & 106 & 3,2736 & 2,11360 & & & \\
\hline ST3 & VA & 106 & 5,2358 & 3,00651 & 2,230 & 210 & ,027 \\
\hline & VC & 106 & 4,3585 & 2,71570 & & & \\
\hline ST & VA & 106 & 16,3019 & 5,90588 & 4,627 & 210 & ,000 \\
\hline & VC & 106 & 12,6792 & 5,48686 & & & \\
\hline
\end{tabular}


- El alcohólico tiende a aislarse y ser individualista. Sus puntuaciones son más bajas en Dependencia de la Recompensa ( $\downarrow R D)$ y sus subescalas de Desapego e Independencia, lo que indica una tendencia a estar solos y aislados de la gente ( $\downarrow$ RD3) y al individualismo ( $\downarrow R D 4)$.

- El alcohólico tiene un menor control de su conducta, hábitos negativos y tiende a proyectar la culpa en los demás ( $\downarrow C$ ) las puntuaciones bajas en autodirección se observan: con Intolerancia Social ( $\downarrow$ C1), más Insensibles ( $\downarrow$ C2), Vengativos ( $\downarrow C 4)$ y Oportunistas ( $\downarrow C 5)$. En cambio el grupo control tiene mayor control de su conducta y tiende a hábitos sanos.

- El varón alcohólico es menos cooperativo ( $\downarrow$ C) que el control: con Intolerancia Social ( $\downarrow C 1)$, más Insensibles ( $\downarrow C 2)$, Vengativos ( $\downarrow C 4)$ y Oportunistas $(\downarrow C 5)$.

En segundo lugar, las diferencias más significativas que encontramos entre el grupo de mujeres alcohólicas y mujeres control (tabla IV):
DIFERENCIAS ENTRE MUJERES ALCOHÓLICAS Y MUJERES CONTROLES

- La mujer alcohólica tiende a anticipar la ansiedad y a ser pesimista, tienen puntuaciones más altas en Evitación del Riesgo ( $\uparrow \mathrm{HA}$ ): en Ansiedad Anticipatoria y pesimismo ( $\uparrow \mathrm{HA} 1$ ), lo que las describe como más neuróticas que las mujeres controles. La mujer control tiende a ser optimista y con energía.

- La mujer alcohólica tiende un menor control de su conducta, hábitos negativos y tiende a proyectar la culpa en los demás, ya que su Autodirección $(\downarrow S)$ es más baja, así se muestra en las subescalas de Proyección de Culpa ( $\downarrow$ S1), de Ausencia de Metas ( $\downarrow S 2)$, de Inercia ( $\downarrow$ S3) y de Malos Hábitos ( $\downarrow$ S5). La tendencia es opuesta a estos resultados en la mujer control.

- La Mujer alcohólica es menos cooperativa que la mujer control ( $\downarrow$ C). Y puntúa bajo en la subescala Oportunismo ( $\downarrow$ C5).

TABLA IV. DIFERENCIA DE MEDIAS ENTRE MUJERES ALCOHOLICASY MUJERES CONTROLES

\begin{tabular}{|c|c|c|c|c|c|c|c|}
\hline & NHIST & $\mathbf{N}$ & Media & Desviación típ. & $\mathbf{T}$ & gl & Sig. (bilateral) \\
\hline \multirow[t]{2}{*}{ HA1 } & MA & 39 & 6,5897 & 2,30238 & 3,507 & 76 & ,001 \\
\hline & $\mathrm{MC}$ & 39 & 4,8205 & 2,15054 & & & \\
\hline \multirow[t]{2}{*}{$\mathrm{HA}$} & MA & 39 & 21,3333 & 4,97009 & 2,681 & 76 & ,009 \\
\hline & MC & 39 & 18,4103 & 4,65504 & & & \\
\hline \multirow[t]{2}{*}{ S1 } & MA & 39 & 3,2564 & 2,13638 & $-4,902$ & 76 &, 000 \\
\hline & $\mathrm{MC}$ & 39 & 5,5385 & 1,97146 & & & \\
\hline \multirow[t]{2}{*}{ S2 } & MA & 39 & 4,0769 & 2,08231 & $-4,556$ & 76 &, 000 \\
\hline & MC & 39 & 6,0769 & 1,78273 & & & \\
\hline \multirow[t]{2}{*}{ S3 } & MA & 39 & 1,4359 & 1,23106 & $-6,044$ & 76 & ,000 \\
\hline & $M C$ & 39 & 3,1795 & 1,31533 & & & \\
\hline \multirow[t]{2}{*}{ S5 } & MA & 39 & 5,3846 & 2,37991 & $-6,610$ & 76 &, 000 \\
\hline & MC & 39 & 8,7436 & 2,09910 & & & \\
\hline \multirow[t]{2}{*}{ S } & MA & 39 & 21,0513 & 6,09119 & $-6,779$ & 76 &, 000 \\
\hline & $\mathrm{MC}$ & 39 & 30,4872 & 6,20217 & & & \\
\hline \multirow[t]{2}{*}{ C5 } & MA & 39 & 5,8205 & 1,29517 & $-3,340$ & 76 & ,001 \\
\hline & $\mathrm{MC}$ & 39 & 6,7436 & 1,14059 & & & \\
\hline \multirow[t]{2}{*}{ ST1 } & MA & 39 & 6,9231 & 2,46432 & 3,549 & 76 & ,001 \\
\hline & $\mathrm{MC}$ & 39 & 4,9487 & 2,44894 & & & \\
\hline \multirow[t]{2}{*}{ ST2 } & MA & 39 & 4,7692 & 2,14552 & 3,727 & 76 & ,000 \\
\hline & $\mathrm{MC}$ & 39 & 3,0513 & 1,91873 & & & \\
\hline \multirow[t]{2}{*}{ ST } & MA & 39 & 16,8718 & 5,29737 & 3,464 & 76 & ,001 \\
\hline & $M C$ & 39 & 12,4615 & 5,92873 & & & \\
\hline
\end{tabular}


También se estudió las diferencias significativas entre los Varones y las Mujeres Controles (tabla V), con el fin de observar de qué manera influye el sexo en los resultados: TROL

DIFERENCIAS ENTRE VARON Y MUJER CON-

- El varón control tiende a ser conservador en el pensamiento y las relaciones sociales, los varones Control tienen puntuaciones más bajas en búsqueda de novedades ( $\downarrow N S)$ : y en sus subescalas Rigidez Estoica ( $\downarrow$ NS1) y Extravagancia ( $\downarrow$ NS3); La mujer control tiende a ser más activa en las relaciones sociales, prefiere iniciar la conversación.
- El varón tiende a tener menos miedos y se siente con más energía. en Evitación del Riesgo ( $\downarrow$ HA): por las subescalas de Confianza ( $\downarrow$ HA2) y de Fatigabilidad ( $\downarrow$ HA4); La mujer control tiende a tener mas miedo a la incertidumbre, y a las situaciones no familiares; se siente con menos energía y se recupera mas despacio

- El varón es menos sensible y considera más importante ser práctico. y en Dependencia de la Recompensa ( $\downarrow R D)$ : por la Insensibilidad ( $\downarrow R D 1)$. La mujer control es más sentimental y comprensiva.

Cuando miramos las diferencias estadísticamente significativas entre los varones alcohólicos y las muje-

\begin{tabular}{|c|c|c|c|c|c|c|c|}
\hline \multicolumn{8}{|c|}{ TABLA V. DIFERENCIA DE MEDIAS ENTRE CONTROLES VARONESY MUJERES } \\
\hline & SEX & $\mathbf{N}$ & Media & Desviación típ. & $\mathbf{T}$ & gl & Sig. (bilateral) \\
\hline \multirow[t]{2}{*}{ NS1 } & VC & 106 & 5,3679 & 2,31486 & $-1,952$ & 143 & ,053 \\
\hline & $\mathrm{MC}$ & 39 & 6,1795 & 1,93135 & & & \\
\hline \multirow{2}{*}{ NS3 } & VC & 106 & 4,2170 & 2,20823 & $-1,901$ & 143 & ,059 \\
\hline & MC & 39 & 4,9744 & 1,88466 & & & \\
\hline \multirow[t]{2}{*}{ HA2 } & VC & 105 & 4,5143 & 1,76567 & $-1,980$ & 142 & ,050 \\
\hline & $M C$ & 39 & 5,1538 & 1,59833 & & & \\
\hline \multirow[t]{2}{*}{ HA4 } & VC & 106 & 2,9245 & 1,94544 & $-2,827$ & 143 &, 005 \\
\hline & $\mathrm{MC}$ & 39 & 3,9744 & 2,08361 & & & \\
\hline \multirow[t]{2}{*}{ HA } & VC & 105 & 15,8190 & 6,30443 & $-2,339$ & 142 &, 021 \\
\hline & $M C$ & 39 & 18,4103 & 4,65504 & & & \\
\hline \multirow[t]{2}{*}{ RD1 } & VC & 106 & 6,9906 & 1,82311 & $-1,989$ & 143 & ,049 \\
\hline & $M C$ & 39 & 7,6410 & 1,51288 & & & \\
\hline \multirow[t]{3}{*}{$\mathrm{RD}$} & VC & 106 & 15,4340 & 3,45038 & $-2,058$ & 143 &, 041 \\
\hline & $\mathrm{MC}$ & 39 & 16,7949 & 3,74292 & & & \\
\hline & SEX & $\mathbf{N}$ & Media & Desviación típ. & $\mathbf{T}$ & GI & Sig. (bilateral) \\
\hline \multirow[t]{2}{*}{ HA1 } & VA & 106 & 5,5660 & 2,59310 & $-2,170$ & 143 &, 032 \\
\hline & MA & 39 & 6,5897 & 2,30238 & & & \\
\hline \multirow[t]{2}{*}{ HA2 } & VA & 106 & 4,5377 & 1,64563 & $-3,706$ & 143 &, 000 \\
\hline & MA & 39 & 5,6410 & 1,42325 & & & \\
\hline \multirow[t]{2}{*}{ HA4 } & VA & 106 & 4,0000 & 2,12020 & $-2,004$ & 143 &, 047 \\
\hline & MA & 39 & 4,7692 & 1,84193 & & & \\
\hline \multirow[t]{2}{*}{$\mathrm{HA}$} & VA & 106 & 18,5849 & 6,06452 & $-2,533$ & 143 & ,012 \\
\hline & MA & 39 & 21,3333 & 4,97009 & & & \\
\hline \multirow[t]{2}{*}{ RD4 } & VA & 106 & 2,7925 & 1,35729 & $-2,521$ & 143 & ,013 \\
\hline & MA & 39 & 3,4103 & 1,16343 & & & \\
\hline \multirow[t]{2}{*}{$\mathrm{RD}$} & VA & 106 & 14,1038 & 3,86419 & $-2,666$ & 143 & ,009 \\
\hline & MA & 39 & 16,0513 & 3,99966 & & & \\
\hline \multirow[t]{2}{*}{ S3 } & VA & 106 & 2,2170 & 1,40750 & 3,060 & 143 & ,003 \\
\hline & MA & 39 & 1,4359 & 1,23106 & & & \\
\hline \multirow[t]{2}{*}{ C3 } & VA & 106 & 4,7830 & 1,65618 & $-3,955$ & 143 & ,000 \\
\hline & MA & 39 & 5,9231 & 1,15587 & & & \\
\hline \multirow[t]{2}{*}{ C4 } & VA & 106 & 7,0000 & 2,57460 & $-2,192$ & 143 & ,030 \\
\hline & MA & 39 & 8,0256 & 2,27674 & & & \\
\hline \multirow[t]{2}{*}{ C } & VA & 105 & 26,6286 & 6,32799 & $-3,254$ & 142 & ,001 \\
\hline & MA & 39 & 30,2564 & 4,73908 & & & \\
\hline
\end{tabular}


res alcohólicas (tabla V), podemos ver que en el Temperamento, las diferencias son muy similares a las encontradas entre ambos grupos control:

\section{DIFERENCIAS ENTRE MUJERES ALCOHÓLICAS} Y VARONES ALCOHÓLICOS

- Las mujeres alcohólicas tienen puntuaciones más altas en Evitación del Riesgo ( $\uparrow \mathrm{HA}$ ) que los varones alcohólicos; lo que indicaría que tienden a ser más pesimistas y preocupadas, tienen más miedo a la incertidumbre y se suelen fatigar con más facilidad. El varón alcohólico es despreocupado, prefiere el riesgo, cree tener más energía y responde impulsivamente a estímulos aversivos.

- La mujer alcohólica tiende a ser más dependiente de los otros y puntúa más alto en Dependencia de la Recompensa ( $\uparrow \mathrm{RD}$ ). El varón alcohólico es individualista y no reflexiona sobre su conducta puntúan más bajo que las mujeres en Independencia ( $\downarrow R D 4)$ : lo que indica tienden a actuar de forma individual, sin preocuparse por la aprobación de los demás.

- Los varones alcohólicos puntúan más alto en Autodirección ( $\uparrow S$ ) a costa de la subescala Con Recursos ( $\uparrow 33$ ), que indica una mayor confianza en la resolución de problemas que las mujeres alcohólicas. Estas tienden a esperar a que les solucionen los problemas

- Las Mujeres Alcohólicas son más colaboradoras y tienen más facilidad para perdonar que los Varones Alcohólicos, que tienen más bajas puntuaciones en Cooperación ( $\downarrow C$ ) y en sus subescalas: de Egoísmo ( $\downarrow$ C3) (se encuentra más centrados en sus propios intereses), y de Venganza ( $\downarrow$ C4) (son más resentidos).

\section{DISCUSIÓN DE RESULTADOS}

En primer lugar, queremos señalar una limitación a destacar en el presente estudio:

El periodo de abstinencia alcohólica antes de la administración de los cuestionarios del TCl es breve (4 semanas), y este hecho puede suponer un sesgo, ya que hay que tener en cuenta que las medidas de los rasgos de la personalidad pueden estar influidas aún por la intoxicación crónica de alcohol reciente, y por el estado emocional, la baja autoestima, o los conflictos que el sujeto tiene que abordar al regresar a la abstinencia. Tras varios meses de tratamiento, es posible que las respuestas obtenidas no se vean contaminadas por estos estados y reflejen mejor la personalidad premórbida. Por tanto, este estudio debería ser revisa- do después de pasados varios meses de tratamiento, para confirmar si se han producido cambios significativos en las medidas de la personalidad o si estos rasgos se han mantenido estables. Sin embargo el hecho de estar sometidos a psicoterapia y a tratamiento psicofarmacológico la mayor parte de los alcohólicos de la muestra, podría influir también a largo plazo en esos posibles cambios.

En segundo lugar, en cuanto al análisis de los resultados:

Estos datos confirmarían los estudios citados anteriormente (Kampov-Polevoy et al., 1998; Basiaux et al.,2001) que indicaban que lo característico de la personalidad del alcohólico, sería la alta Búsqueda de Novedades ( $\uparrow N S$ ), y las bajas Evitación del Riesgo ( $\downarrow$ HA) y Autodirección ( $\downarrow S)$. De manera que nuestros resultados pueden ser una evidencia adicional.

Por otra parte, la suma de una baja Autodirección $(\downarrow S)$ y una baja Cooperación $(\downarrow C)$ fue descrita como un indicador de presencia de trastorno de la personalidad (Cloninger et al.,1995), lo que apuntaría a una mayor presencia de estos trastornos en nuestra muestra de población clínica que en los controles.

Apoya esta idea el hecho de que tanto el grupo de mujeres como el de hombres alcohólicos presentan puntuaciones medias en Autodirección ( $\mathrm{S} v=23,4$ y $\mathrm{Sm}=21,05$ en varones y mujeres respectivamente) y Cooperación ( $\mathrm{Cv}=21$ y $\mathrm{Cm}=30,25)$ inferiores a la media encontrada en población general en la el estudio de Mateos y De la Gándara (2001). Si atendemos a la Autodirección, el riesgo de presentar un trastorno de la personalidad es mayor para las mujeres, sin embargo si atendemos a la relación Autodirección/Cooperación, si bien las medias encontradas en ambos grupos de alcohólicos describen un Carácter Inmaduro $(S+C<58)$, y por tanto una tendencia a la inestabilidad, en las mujeres esta matizada por una mayor Cooperación (C).

Si observamos las puntuaciones medias de ambos grupos, podemos decir que la tipología temperamental de los Varones alcohólicos en su conjunto se corresponde con el tipo Explosivo-Borderline de Cloninger (NS+; HA+; RD-), mientras que la de las mujeres alcohólicas es del tipo Aventurero-Antisocial del mismo autor (NS+; HA-; RD-), ambas en el espectro del Clúster $B$ del DSM. No se ha estudiado la proporción de estas tipologías en cada subgrupo.

Por otra parte una Evitación del Riesgo alta (HAv= 18,5 y HAm $=21,33$ ) apunta a la presencia de trastornos de ansiedad, y la combinación de alta Evitación del Riesgo, baja Autodirección y baja Cooperación ( $\uparrow$ $H A+\downarrow S+\downarrow C)$ es el patrón de los trastornos depresivos, características encontradas en nuestra muestra.

En cuanto a la idea de descartar o afianzar la idea de la existencia de unos rasgos característicos de las personalidades alcohólicas, las diferencias encontradas 
entre ambos grupos indican que pudieran separarse los perfiles de personalidad de nuestros Alcohólicos y la Población No Clínica estudiada, si bien queremos insistir en la idea de que no podemos valorar la influencia directa del alcohol en estos sujetos, por acción directa y permanente o a largo plazo del alcohol sobre la estructura cerebral o por las necesidades de readaptación al estado de abstinencia un mes después de abandonar su consumo.

Estas diferencias se caracterizarían en cuanto a las dimensiones del temperamento en:

$1^{\circ}$ Una mayor Búsqueda de Novedades (NS): ante situaciones de apatía, aburrimiento y tristeza tienen conductas exploratorias cuya finalidad es alcanzar premios inmediatos y evitar los castigos, frecuentemente a través del consumo de alcohol, buscando inmediatamente el premio.

Son más impulsivos que los controles, actuando sin que medie la reflexión y cambiando bruscamente de interés. También destaca su tendencia al desorden, la dificultad para asumir normas y la tendencia a transgredirlas, sobre todo cuando saben que no van a ser sorprendidos. Son ambiguos, y saben improvisar, mentir y que les crean.

Estos rasgos tienen una implicación para el tratamiento de estos pacientes. Desde un punto de vista biológico, la Búsqueda de Novedades esta regulada por las vías dopaminérgicas mesolímbicas. La comprensión de este sustrato biológico puede permitir estrategias psicofarmacológicas (Bloqueo dopaminérgico, o endorfínico, o mediante otras vías indirectas que regulen la actividad de este módulo neurobiológico) que favorezcan una mayor contención, disminuir la impulsividad y aumentar la tolerancia a la frustración.

Desde un punto de vista psico-social, el paso al acto implica la ausencia de un espacio mental que permita vivir las situaciones de tensión o frustración para trasformarlas en un proceso simbólico, en el que los sentimientos den lugar primero a palabras capaces de describirlos y comprenderlos-aprenderlos y después para planificar estrategias que permitan la esperanza de cambiarlos y en último extremo planificar y poner en marcha acciones para el cambio. Para aumentar la tolerancia a la frustración, entonces tendremos que establecer una estructura de contención que permita que el paciente no pueda evitar fácilmente, pero que simultáneamente pueda depositar su responsabilidad y la capacidad de espera en un primer momento en una figura parental, que si es vivida de forma no persecutoria, le permitirá introyectarla en su función paterna y con ello las normas necesarias para cuidarse, evitando situaciones de riesgo, ganando en autocontrol emocional, afrontando situaciones nuevas y planificando las tareas diarias. $2^{\circ}$ Una mayor en Evitación del Riesgo (HA), sobretodo en el caso de las mujeres alcohólicas: Tienden a evitar más las situaciones ansiógenas, frustrantes o dolorosas que los controles, lo que implica una inclinación a responder a estímulos aversivos con conductas de evitación. Son personas tensas y preocupadas en situaciones nuevas, con temor a la incertidumbre, que se fatigan antes y se recuperan despacio de las tensiones.

Desde el punto de vista biológico, esta dimensión esta regulada por el sistema septohipocampico y las proyecciones serotoninérgicas de los núcleos del rafe, por tanto regulado por la Serotonina, si bien las Benzodiazepinas también pueden reducir las conductas evitativas mediante la inhibición a través del GABA, de las proyecciones serotoninérgicas de los núcleos del rafe. El conocimiento de estas implicaciones nuevamente nos puede brindar estrategias de intervención psicofamacológica.

Desde un punto de vista psicosocial, sobre todo en el caso de las mujeres alcohólicas, en las orientaciones para el tratamiento, deberíamos tener en cuenta que un objetivo primordial es reparar su autoestima dañada, su autoconcepto, y que al descubrir el valor de habilidades que poseen, pero que no aprecian, o del desarrollo de otras nuevas, puedan ser menos sensibles a potenciales peligros, y puedan enfrentarse progresivamente a situaciones que teman no controlar o les produzcan temor, mejorando así su capacidad para la resolución de problemas.

$3^{\circ}$ Paradójicamente tienen una Menor Dependencia de la Recompensa (RD): que indica una tendencia a la soledad, a un mayor desapego y a actuar de forma individualista, sin preocuparse por si son aprobados socialmente o no. Este hecho quizás venga determinado por desarraigo social o una tendencia a proyectar sus dificultades que les lleve a sentirse dañados por la mirada de los demás.

En cuanto al desarraigo social, o la propia imagen social dañada, que puede estar en el trasfondo de estos resultados, entendemos que un objetivo con los pacientes alcohólicos debe ser facilitar su integración efectiva en distintos grupos sociales, para que puedan actuar e implicase afectivamente como parte de esos grupos. Desde un punto biológico, en la base de esta dimensión están las vías noradrenégicas que regulan la estabilidad.

\section{Desde el punto de vista de las dimensiones del} carácter:

Presentan una baja Auto-dirección en comparación con el grupo control, que implica una menor habilidad en el control, regulación y adaptación de su comportamiento, no ajustándose a las situaciones según metas y valores escogidos individualmente. Su imagen del Self es autopercibida como poco inte- 
grada, teniendo dificultad para dar sentido a su vida, y viéndose como desorganizados y con reacciones impulsivas.

También tienen una mayor tendencia a culpabilizarse, sintiéndose influenciados por los demás y pocas veces libres para elegir. La culpa es un factor clave en el alcohólico, se debe enseñar a manejar, para que no impida la evolución positiva.

Por fin, puntúan alto en Ausencia de metas, y en Malos Hábitos, lo que indicaría que no se gustan por su escasa fuerza de voluntad y por sus imperfecciones y se sienten incapaces para dar sentido a su vida.

Por tanto, otro objetivo importante a tener en cuenta en el tratamiento, sería que el paciente alcohólico pueda reconocer y mejorar su autoconcepto, y se acepte a sí mismo. Esto permitiría que valorase sus capacidades, aptitudes y que se sintiera más compensado emocionalmente. Este proceso implica, como se dijo anteriormente, rescatar las figuras parentales, elaborar duelos, y a veces identificarse con nuevas figuras e introyectarlas. También afrontar situaciones temidas y ganar en competencia.

Como hacíamos referencia anteriormente, creemos que es necesario que el paciente tenga una planificación de su vida cotidiana, con metas específicas, y que ciertos hábitos no favorables para su salud o para una situación de riesgo de recaída sean modificados y sustituidos por otros más sanos.

La menor tendencia a la Cooperación: procede de una mayor Intolerancia Social, insensibilidad, egocentrismo y resentimiento. El trabajo en grupos de psicoterapia o situaciones sociales con pacientes mejora esa intolerancia social y esa incapacidad para integrarse socialmente, cuando pueden recibir la propia imagen devuelta por los otros miembros del grupo en un clima de confianza y seguridad, haciéndoles mas sensibles y afectivos.

Para terminar, señalar que en una observación global de las tablas, Ilama la atención que los Varonas Alcohólicos, se diferenciaban en todas las escalas del $\mathrm{TCl}$, excepto la Persistencia (P), y en la mayoría de las subescalas, mostrándose más necesitados de estímulos nuevos y neuróticos, pero sorprendentemente menos dependientes de la recompensa. También la Autodirección y cooperación eran menores, lo que les señala como un grupo más patológico que los controles. La Autodirección es una escala que no este bien definida para su uso clínico, al menos de momento, y la única relación que Cloninger (1995) encontró entre ST y la patología, fue con los trastornos esquizotípicos de la personalidad. En nuestro estudio, el grupo de varones y mujeres alcohólicas puntuaron más alto que los controles en esta dimensión.

Las diferencias entre las mujeres son similares a las encontradas entre los varones, pero no aparecen en Búsqueda de Novedades, en Dependencia de la Recompensa ni en Cooperación, aspectos en los que ambos grupos son iguales.

Este hecho se confirma cuando tratamos de ver las diferencias entre hombres y mujeres en población no clínica: las mujeres, en general, son más neuróticas y dependientes que los varones y por tanto más conservadoras, pero más cooperativas (este último extremo, curiosamente, no hemos podido comprobarlo en esta comparación de medias).

Por fin, las diferencias entre varones y mujeres alcohólicas son similares a las encontradas entre los controles, si bien en este caso ellas son más cooperadoras que los varones.

\section{REFERENCIAS}

Basiaux, P., Le Bon, O., Dramaix, M., Massat I.; Souery, D., Mendlewicz, J., Pelc, I. y Verbanck, P. (2001). Temperament and Character Inventory (TCI) personality profile and sub-typing in alcoholics patients: a controlled study; Alcohol Alcohol, 36, 584-587.

Belloch, A. y Sandín, B. (1995). Manual de psicopatología. Madrid: Ed. MacGraw Hill.

Casariego, C., Morera, A., Henry, M., Valenciano, R., Bello, G. y Cuesta, J. (1995). Psicopatología en alcohólicos en desintoxicación: relación con variables toxicológicas y sociodemográficas. Adicciones, 7, 25-30.

Cloninger, C.R., Przybeck, T.R. y Svrakic, D.M. (1993). A psychobiological model of temperament and character. Arch. Gen. Psychiatry, 50, 975-990.

Cloninger, C.R., Sigvardsson, S., Svrakic, D.M. y Przybeck, T.R. (1995). Personality antecedents of alcoholism in a national area probability sample. Eur. Arch. Psychiatry Clin. Neurosci., 245, 239-244.

Cloninger, C.R. y Svrakic, D.M. (1997). Integrative psychobiological approach to psychiatric assessment and treatment. Psychiatry, 60, 120-141.

Cloninger, C.R. (1994). Temperament and personality. Curr Opin Neurobiol, 4, 266-273.

Cloninger, C.R. (1987). A systematic method for clinical description and classification of personality variants. Arch. Gen. Psychiatry, 44, 573-5888.

Fallgatter, A.J., Weijers, H.G., Wiesbeck, G.A., Boening, J. y Strik, W.K. (1998). Event-related correlates of response suppression as indicators of novelty seeking in alcoholics. Alcohol alcohol, 33, 475-481.

Gutiérrez, F., Torrens, M., Martín-Santos, R., Sangorrin, J., Pérez, G. y Salamero, M. (2001). Psychometric properties of the Temperament and characternality using the Temperament and Character Inventory (TCl) 
questionnaire in a Spanish psychiatric population. Acta Psychiatr Scand, 103, 122-128.

Howard, M.O., Kivlahan, D. y Walker, R.D. (1997). Cloninger's tridimensional theory of personality and psychopathology: applications to substanceuse disorders. J. Subst Abuse, 58, 48-66.

Johnson, B.A., Cloninger, C.R., Roache, J.D., Bordnick, P.S. y Ruiz, P. (2000). Age of onset as a discriminator between alcoholic subtypes in a treatment-seeking outpatient population. J. Adict, 9, 17-27.

Kampov-Polevoy, A.B., Garbutt, J.C., Davis, C.E. y Janowsky, D.S. (1998). Preference for higher sugar concentrations and Tridimensional Personality Questionnaire scores in alcoholic and nonalcoholic men. Alcohol Clin. Exp. Res, 22, 610-614.

Kitamura, T., Kijma, N., Sakamoto, S., Tomoda, A., Suzuki, N. y Kazama, Y. (1999). Correlates of problem drinking among young Japanese woman: personality and early experiences. Compr. Psychiatry., 40, 108-14.

Mateos, M. y De la Gandara, J.J. (2001). Temperamento carácter y personalidad. Guía práctica de corrección y evaluación del TCl (Inventario de Temperamento y Carácter, de Robert C. Cloninger). Madrid: SMC. GlaxoSmithKline.

Mateos, M. y De la Gándara (2001): Aplicación Informática para el Tratamiento de Datos, Corrección del Test y Emisión de Informes. Madrid: SMC. GlaxoSmithKline.

Mateos, M., Ruiz, J.M. y De la Gándara, J.J.. Temperamento, carácter, impulsividad: una aproximación al modelo psicobiológico de personalidad de Cloninger. $2^{\circ}$ congreso Virtual de Psiquiatría 10m1 conf 5. Recuperado el 15 de Marzo del 2003 de http://psiquiatria.com.

Mateos, M., Sanz, B., Palomo, J., García, C., Merino, S. y De la Gándara, J.J.. Perdida de control en el uso de sustancias y $\mathrm{TCl} .2^{\circ}$ congreso Virtual de Psiquiatría 10m1 conf 4. Recuperado el 15 de Marzo del 2004 de http://psiquiatria.com.

Meszaros, K., Willinger, V., Fischer, G., Schonbeck, G. y Aschaner, H.N. 1996. The tridimensional personality model: influencing variables in a sample of detoxified alcohol dependents. European Fluvoxamines in Alcoholism Study Group. Compr. Psychiatry. 37, 109-14.

Modesto-lowe, V. y Kranzler, H.L. (1999). Diagnosis and treatment of alcohol-dependent patients with comorbid psychiatric disorders. Alcohol Res Health, 23, 144-149.

Pomerleau, C.S., Pomerleau, D.E., Flessland, K.A. y Basson, M.S. (1992). Relationship of Tridimensional Personality Questionannaire scores and snoking variables in female and male smokers; J. Subst Abuse, 4, 143-54.

Sander, T., Harms, H., Dufen, P., Kuhn, S., Hoeche, M., Lesch, K.P., Schmidt, LG. y Rommelspacher, H. (1998). Serotonin transporter gene variants in alcoholdependent subjects with dissocial personality disorder. Biol. Psychiatry 43, 908-1012.

Schmidt, LG., Dufeu, P., Kuhn, S. y Rommelspacher, H. (1994). Relapse prevention in alcoholics with an anticraving drug treatment: first results of the Berlin study. Pharmacopsychiatry 27, 21-3.

Thome, J., Weijers, H.G., Wiesbeck, G.A., Sian, J., Nara, K., Boening, J. y Riederer, P. (1999). Dopamine D3 receptor gene polymorphism and dependence relation to personality rating. Psychiatr Genet, 9, 713-20.

Weijers, H.G., Wiesbeck, G.A. y Boening, J. (1999). El temperamento y rasgos de carácter, así como modelos de rasgo hombres alcohólicos y controles.; J. Subst Abuse, 70, 998-1008.

Willis T.A., Vaccaro D. y Mcnamara G.A. (1994). Novelty seeking, risk taking, and related constructs as predictors of adolescent substance use: an application of Cloninger's theory. J. Subst Abuse, 6, 1-20. 
